\title{
Theoretical study of boundary lubrication
}

\author{
A. Buldum and S. Ciraci \\ Department of Physics, Bilkent University, Bilkent 06533, Ankara, Turkey
}

(Received 7 October 1998)

\begin{abstract}
We analyzed the dynamics of xenon atoms as lubricant between two Ni(110) slabs in relative motion. Atomic simulations are carried out by using classical molecular dynamics with realistic empirical potentials, where nickel as well as xenon atoms are relaxed. The resistance of the xenon layer against the loading force is examined and critical forces are determined to destroy the lubricant layer at different coverages. The relative motion of slabs in the lateral direction is investigated under constant normal force as a function of coverage ranging from zero to the monolayer xenon. Important lubrication properties of xenon atoms are analyzed by calculating the variation of potential energy, lateral force, and local hydrodynamic pressure. It is predicted that the corrugation of the potential energy associated with the sliding has a minimum value at submonolayer coverage. A phononic energy dissipation mechanism together with the theoretical analysis is proposed.
\end{abstract} [S0163-1829(99)08623-3]

\section{INTRODUCTION}

Friction and tribology compose an old as well as important subject. ${ }^{1}$ Earlier studies have shown that the understanding of several complex phenomena taking place in friction, such as electronic and phononic energy dissipation, wear, structural phase transitions, etc., can be provided by atomic scale investigations. Recent progress in the atomic force microscopy ${ }^{2,3}$ (AFM) has made precise and atomic scale force measurements possible. The valuable data gained from these measurements have been combined with the extensive atomic simulations $^{4-9}$ and $a b$ initio force calculations ${ }^{10}$ to investigate friction on the atomic scale. The boundary lubrication that involves lubricant atoms or molecules between surfaces in relative motion becomes even more complex. The coverage of the lubricant can vary from submonolayer to a few layers, i.e, $0<\Theta<n$, and prevents the moving objects from direct contact. Moreover, some lubricant atoms or molecules shield the interaction between sliding surfaces and weaken the corrugation of the adhesive energy. These effects are combined to reduce the friction coefficient. For a given type of lubricant, the friction coefficient depends mainly on the coverage $\Theta$, velocity of relative motion $\mathrm{v}$, and normal force distribution $F_{N}$. Experiments performed by using a surface force apparatus showed different regimes (i.e., stickslip and steady sliding motion) depending on the velocity $\mathrm{V}$ of the moving object. ${ }^{11}$ By increasing $\mathrm{V}$ over the critical velocity the stick-slip motion is transformed into steady-state sliding. ${ }^{11-13}$

The lubricant being an atom or molecule is a critical ingredient in the boundary lubrication. An efficient lubricant is expected to screen the strong interaction between the atoms of the surfaces, in relative motion. If these were bare metal surfaces, the interatomic interaction would be very strong. For example the adhesive energy for a metal atom between two metal surfaces is $\sim 1-3 \mathrm{eV}$ and the maximum attractive force $\sim 1-2 \mathrm{nN}$. Under this strong interaction two surfaces normally stick to each other if they are clean and atomically flat. Certain atoms or molecules located between two surfaces (for example the inert-gas atoms) reduce this interaction. If both surfaces are covered by lubricant such as Xe atoms for $\Theta \sim 2$, relative motion takes place between lubricant surfaces where the interatomic interaction is significantly weak. For $\Theta \sim 2-3$ the state of the lubricant between the surfaces is crucial. Structural phase transitions or, in certain conditions, the "chaotic" behavior of lubricant layers during the relative motion of the surfaces and the effects of other parameters such as $\mathrm{v}, F_{N}$, and the area of contact $A$ in the relative motion are of interest. The lubricant atoms moving on the metal surfaces can excite electron-hole pairs and phonons which dissipate mechanical energy of the motion into heat.

The atomic configuration of the lubricant atoms between the moving surfaces is essential for investigation of the boundary lubrication. While this region is not directly probed, important information can be obtained from the atomic simulations. In this work, we performed atomic simulations of $\mathrm{Xe}$ atoms with $\Theta \leqslant 1$ which play the role of lubricant between two $\mathrm{Ni}(110)$ slabs in relative motion. We investigate two cases for different coverages, $0 \leqslant \Theta \leqslant 1$. In the first case, one Ni slab is pressed towards the other one in the vertical direction until $F_{N}$ becomes very large. This way, the behavior of the lubricant layer under large pressure $p$ $=F_{N} / A$ is analyzed. In the second case, one of the slabs is moved laterally relative to the other one under a constant normal force $F_{N}$. While the slabs are pressed by a constant normal force in the course of their lateral relative motion, $\mathrm{Ni}$ as well as Xe atoms are fully relaxed. In this respect, the present simulation provides valuable data for further theoretical studies. Here $\Theta=0$ corresponds to the dry sliding friction and involves irreversible deformation (or atomic rearrangements), whereas $\Theta>0$ represents the boundary lubrication. We examine the variation of the total potential energy $V_{T}$, lateral force $F_{L}$, and the hydrostatic pressure with the displacement and examine the atomic motion. We discuss also a phononic mechanism of energy dissipation in the boundary lubrication.

\section{METHOD}

We performed molecular dynamics calculations by using empirical potentials. Two objects, one moving with a veloc- 


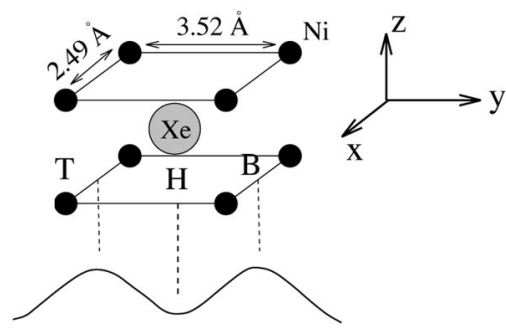

FIG. 1. The unit cell of the moving (upper) and resting (lower) $\mathrm{Ni}(110)$ slabs with the Xe atom between them. The $y$ axis is taken parallel to the [001] direction. The upper slab is at the top site relative to the lower one. Xe atoms are at the hollow site. $T, H$, and $B$ indicate top, hollow, and bridge site positions relative to the lower slab. The lower curve schematically describes the corrugation of the potential energy.

ity $\mathrm{V}$, relative to the other one, are $\mathrm{Ni}(110)$ slabs; each slab has $8 \mathrm{Ni}(110)$ layers and each layer contains 140 unit cells and hence 140 atoms. Periodic boundary conditions are used in the lateral (or $x y$ ) plane described in Fig. 1. The perpendicular or lateral motion of the first slabs is controlled by moving the topmost two layers, but keeping the lowest two layers of the second slab fixed. The rest of the atoms, which we called dynamic atoms ( $\mathrm{Ni}$, as well as $\mathrm{Xe}$ atoms), are relaxed so that the net force acting on each dynamic atoms is diminished. We treated different values of Xe coverages: $\Theta$ $=0,0.64,0.84$, and 1.0 corresponding to $0,45,59$, and 70 $\mathrm{Xe}$ atoms between the $\mathrm{Ni}(110)$ slabs. The Xe-Xe interaction is expressed in terms of Lennard-Jones potential

$$
U_{i j}\left(r_{i j}\right)=\epsilon\left[\left(\frac{r_{0}}{r_{i j}}\right)^{12}-2\left(\frac{r_{0}}{r_{i j}}\right)^{6}\right]
$$

where $r_{i j}=\left|\mathbf{r}_{i}-\mathbf{r}_{j}\right|, \mathbf{r}_{i}$ and $\mathbf{r}_{j}$ denoting the positions vectors of atoms $i$ and $j$, respectively. The parameters $r_{0}=4.36 \AA$ and $\epsilon=0.024215 \mathrm{eV} .{ }^{14}$ Similarly, the interaction between $\mathrm{Xe}-\mathrm{Ni}$ atoms is expressed with a similar potential ${ }^{15}$ with different parameters $r_{0}=3.27 \AA$ and $\epsilon=0.03413 \mathrm{eV}$. The interaction between Ni-Ni atoms is represented by the embedded atom potential. ${ }^{16}$ The coordinate system of the motion, the orientation of $\mathrm{Ni}(110)$ slabs, and their primitive unit cell, with lattice parameters $a=3.52 \AA, b=2.49 \AA$, are illustrated in Fig. 1.

In the perpendicular approach (along the $z$ direction in Fig. 1), one slab is left to descend towards the other from the $h_{0}=6.0 \AA$ in the $\Theta=0$ case and from $h_{0}=8.8 \AA$ in the $\Theta \neq 0$ case in increments of $\Delta d=0.05 \AA$. In the lateral motion of the slab along the [001] direction (or the $y$ direction) that is parallel to the (110) surfaces of both slab, one slab is displaced in steps of $\Delta s=0.05 \AA$ under the constant normal force $F_{N}=0.03 \mathrm{eV} /$ atom $(=0.048 \mathrm{nN} /$ atom $)$. During each time step $\left(\Delta t=10^{-15} \mathrm{sec}\right)$ all dynamic atoms are let to move under the forces acting on them, but they are thermalized to $4 \mathrm{~K}$ after each $\Delta t$ by rescaling their velocities. Between two consecutive displacements (i.e., increments of $\Delta d$ or $\Delta s$ ) all the dynamic atoms are relaxed for 2000 time steps in both perpendicular and lateral motion. Accordingly the lateral speed $v$ can be taken as $2.5 \mathrm{~m} / \mathrm{sec}$. The total potential energy $V_{T}$ is calculated by averaging 1500 time steps follow-
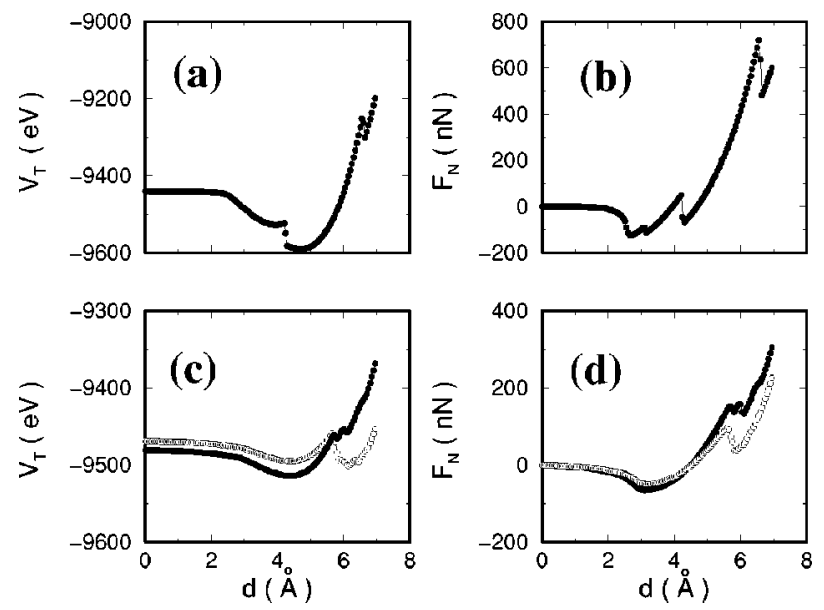

FIG. 2. Total potential energy $V_{T}(d)$ and normal forces $F_{N}(d)$ in the case of pressing the upper slab towards the lower one with different $\Theta$ values. Panels (a) and (b) are for $\Theta=0$. Panels (c) and (d) are for $\Theta=0.64$ (open circles) and $\Theta=0.84$ (solid circles). $d$ is the perpendicular displacement of the upper slab toward the lower one starting at a height $h_{0}$ explained in the text.

ing the first 500 time steps. After $n$ displacement steps, the perpendicular and lateral displacements are $d=n \Delta d$ and $s$ $=n \Delta s$, respectively.

\section{PRESSING}

By displacing the upper slab towards the lower one from the height $h_{0}$, the total potential energy $V_{T}$ is first lowered owing to the attractive interaction. Initially, the bottom surface atoms of the upper slab face the atoms at the surface of the lower slab; it is denoted as the top site $(T)$ registry. Upon further approach $V_{T}$ passes through a minimum and increases due to the repulsive interaction at small separation. The variation of $V_{T}(d)$ and the perpendicular force $F_{N}(d)$ is shown for various values of $\Theta$ in Fig. 2. Figures 2(a) and 2(b) correspond to the case $\Theta=0$. At some points $F_{N}$ decreases suddenly. The first sudden change occurs at $d$ $=3.1 \AA$ where the surface atoms of the upper slab jump to the $H$-site registry having relatively lower energy. The second sudden fall of $F_{N}$ at $d=4.3 \AA$ is due to the jump to contact whereby the upper slab and the lower slab change to the bulk registry. The third sudden fall of the force curve $F_{N}$ occurs at $d=7 \AA$ where the ordered structure of the slabs cannot be maintained under such a high load, $F_{N T}$ $>700 \mathrm{nN}$. We note that $F_{N}$ or $F_{N T}$ induced as a result of the displacement of the upper slab towards the lower one is uniaxial along the $z$ axis.

$\mathrm{Xe}$ atoms between the slabs $(\Theta>0)$ make the total potential energy $V_{T}$ relatively shallower. Initially the Xe atoms stay adsorbed on the surface of the lower slab, and they keep their positions until $d=3.0 \AA$. For $d>3 \AA$ they rise towards the upper slab and are stabilized at the middle of the separation between the surfaces of the slabs, since the energy barrier diminishes and two local energy minima for the Xe atoms (each being closer to one slab surface) collapsed to a single minimum at the middle. The Xe atoms, as well as the atoms of the surfaces of the slabs adjacent to the Xe layer, keep this configuration until $d \sim 5.7 \AA$ (where $F_{N}$ 

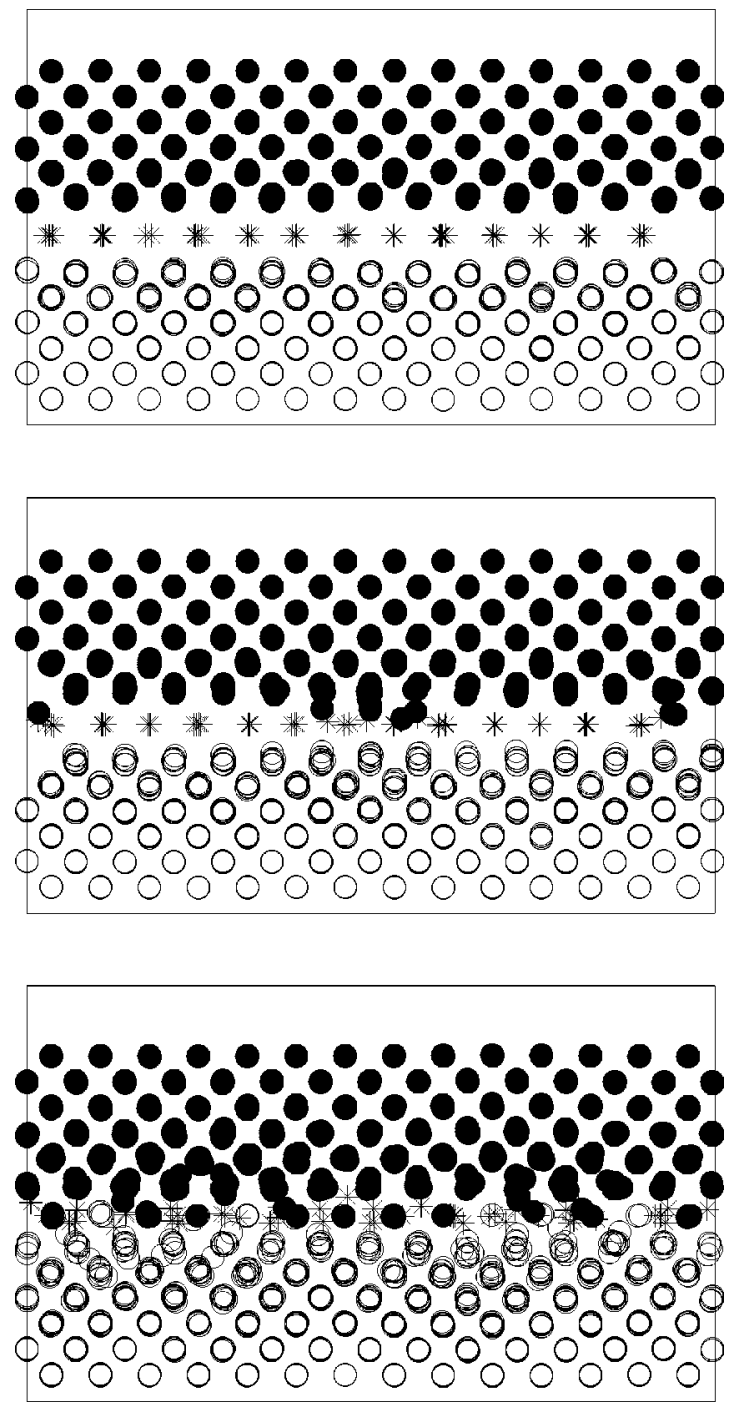

FIG. 3. Snapshots of the slab-lubricant-slab system during pressing are shown for $\Theta=0.64$. The $\mathrm{Ni}$ atoms in the upper (lower) slab are represented by solid (open) circles and the Xe atoms are represented by stars. Panels (a), (b), and (c) correspond to $d=5.6$, 5.7, and $6.0 \AA$ A , respectively.

$\sim 0.7 \mathrm{eV} / \AA$ atom $\sim 1.12 \mathrm{nN} /$ atom) for $\Theta=0.64$. Once the normal force exceeds this critical value the lubrication layer is destroyed, and the Ni atoms of both surfaces establish direct contact. The resistance to the normal load increases with $\Theta$; the critical force $F_{N}=1.6 \mathrm{nN} /$ atom for $\Theta=0.84$, and $F_{N}=3.04 \mathrm{nN} /$ atom for $\Theta=1$. The sudden change in the $F_{N}$ curve is more distinguishable in the $\Theta=0.64$ case, whereby the lubrication layer is destroyed since more $\mathrm{Ni}$ atoms under stress enter into the vacancies in the Xe layer and lower the potential energy. Finally, we note that the spacing between the slabs increases by $\sim 2.8 \AA$ in the presence of Xe atoms. However, this is not so obvious in Fig. 2, illustrating the global minima of $V_{T}$ near $d \sim 4.5 \AA$ for both $\Theta=0$ and $\Theta>0$ cases, since the perpendicular displacement $d$ of the upper slab starts from the height $h_{0}=6$ and $8.8 \AA$ for $\Theta=0$ and $\Theta>0$, respectively. Figure 3 shows how the lubricant layer of $\Theta=0.64$ is destroyed under heavy load. Upon further pressing, more $\mathrm{Ni}$ atoms mix with $\mathrm{Xe}$ atoms and the bottom layers of the upper slab and the top layers of

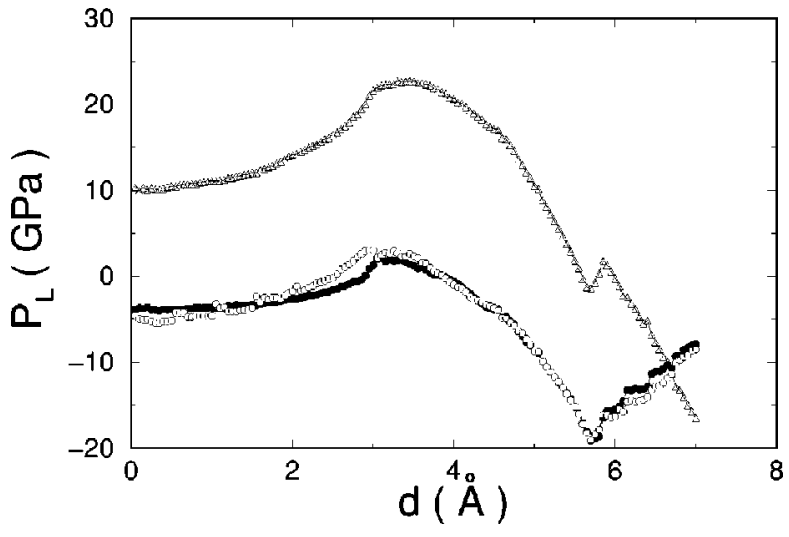

FIG. 4. Layer averaged local hydrostatic pressure values $P_{L}(d)$ for the bottom layer of the upper slab (open circles), the top layer of the lower slab (solid circles), and the lubricant layer (triangles) for $\Theta=0.64$.

the lower slab were deformed.

Beside atomic configuration and atomic forces, other local parameters like local atomic stresses and local hydrostatic pressure are crucial for the study of lubrication. We note that even if the net force on one atom is zero, the hydrostatic pressure may be quite high. The local hydrostatic pressure $P_{i}$ is the trace of the local atomic stress tensor, ${ }^{17}$

$$
P_{i}=(1 / 9)\left(\sigma_{i}^{x x}+\sigma_{i}^{y y}+\sigma_{i}^{z z}\right) .
$$

$P_{i}$ can take positive and negative values; positive values correspond to compressive pressure and negative values correspond to expansive pressure. The average hydrostatic pressure of the layer $L$ is calculated as $P_{L}=\left(1 / n_{L}\right) \sum_{i}^{n_{L}} P_{i}$, where $n_{L}$ denotes the number of atoms in the layer $L$.

The average hydrostatic pressure values $P_{L}$ of the bottom layer of the upper slab, the top layer of the lower slab and the lubrication layer as a function of perpendicular displacement $d$ are shown in Fig. 4. Normally, one expects that the pressure values increase for $\mathrm{Xe}$ and $\mathrm{Ni}$ layers until the destruction of the lubricant layer. But surprisingly, the pressure values first increase and then decrease smoothly. This situation can be understood by analyzing the pressure contours on the $x z$ plane of a slice of the system as shown in Fig. 5. For $d$ $\sim 3.0 \AA$ the pressure intensified initially on the Xe atoms (three Xe atoms on the left and two Xe atoms on the right of the panel have $20 \mathrm{GPa}$ ). By further pressing of the lubricant layer, the high-pressure region switches to the atoms around the vacancy positions. This way $F_{N}$ is distributed to relatively larger cross section and hence the average pressure of the layers decreases. However, this does not mean that the system is going to be more stable, since upon further pressing, the vacancies begin to be filled by the surrounding $\mathrm{Ni}$ atoms. This way $P_{L}$ is decreased further but the lubricant layer is totally destroyed. The behavior of the system under high uniaxial loading force is, however, only qualitatively correct due to the periodic boundary condition and limited size of the system used in atomic simulations.

\section{FRICTION}

The friction of the Ni(110) slab, moving above a similar $\mathrm{Ni}(110)$ slab that is fixed by two bottom layers, is studied for 

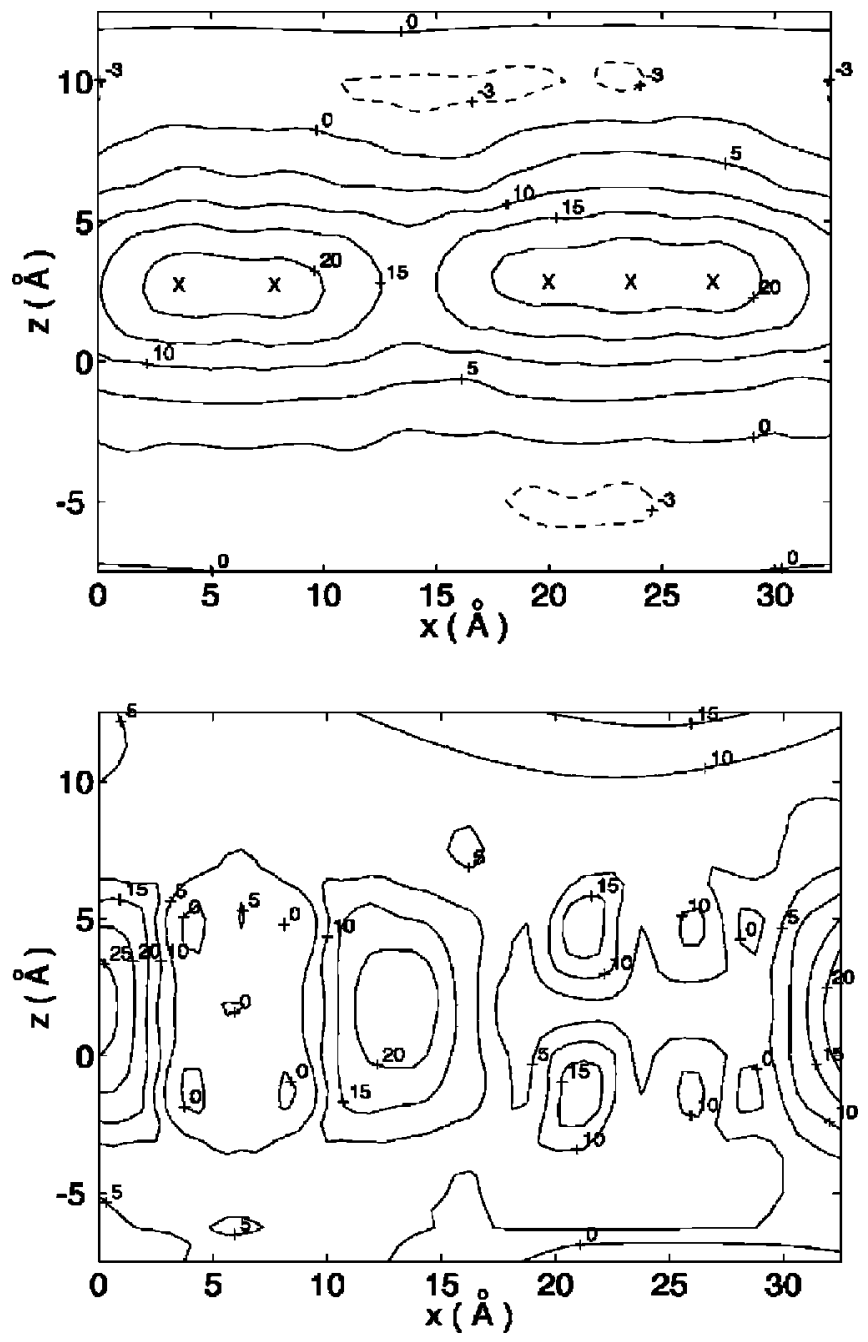

FIG. 5. Contour plots of the local hydrostatic pressure on the $x z$ plane for $\Theta=0.64$. The upper panel corresponds to $d=3.0 \AA$, and lower panel corresponds to $d=5.5 \AA$ just before the destruction of the lubricant layer. Contour values (in units of GPa) are indicated on the plots. Solid and dashed contour lines correspond to the compressive and expansive pressure values, respectively. The positions of $\mathrm{Xe}$ atoms are indicated by $\mathrm{X}$ in the upper panel.

$\Theta=0,0.64,0.84,1.0$ (i.e., full coverage). The translation of the upper slab $s$, that is, $n \Delta s$ after $n$ lateral displacement steps, is made under constant normal load $F_{N}=0.03 \mathrm{eV} / \AA$ atom (or total load $F_{N T}=6.72 \mathrm{nN}$ ). The coverage $\Theta=0$ corresponds to the dry friction. The important difference between $\Theta=0.84$ and 1.0 is that one row and one column of $\mathrm{Xe}$ atoms are missing in the former case. The total potential energy and lateral force variation $V_{T}(s)$ and $F_{L}(s)$ are shown for various values of $\Theta$ in Fig. 6. The relative motion with $\Theta=0$ starts at the $T$ site (see Fig. 1) that is a metastable state. By moving the upper slab the atoms jump the $H$ site of the lower surface. The total potential energy continues to decrease with further displacement and passes through a minimum at $s=1.75 \AA$. This is the minimum energy configuration. Normally, the maximum of $V_{T}(s)$ would appear at the next $T$ site (for $z=3.5 \AA$ and $s \sim 3.5-4.0 \AA$ ). However, before this expected maximum occurs, the total potential energy is lowered by $\sim 30 \mathrm{eV}$ due to a structural transformation from the highly ordered to the disordered state.
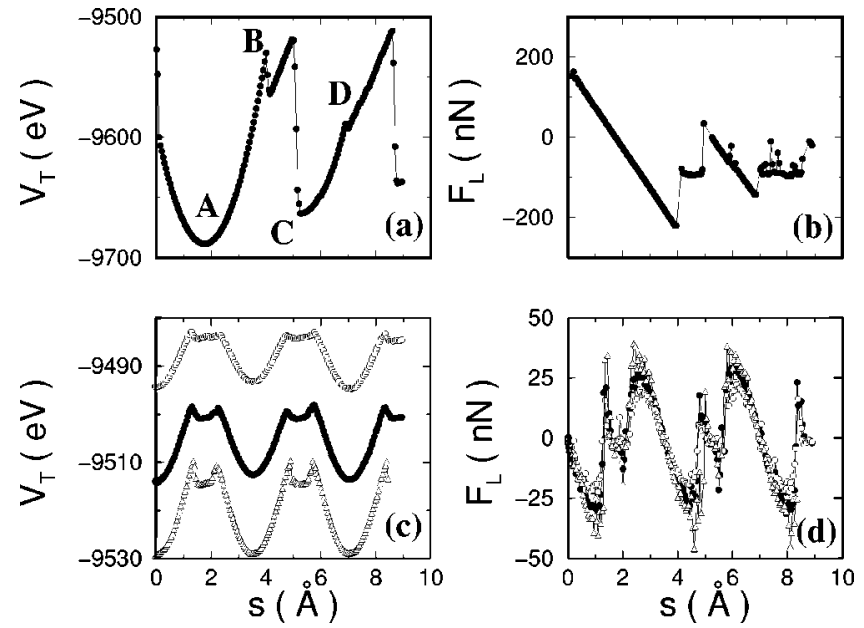

FIG. 6. Potential energy $V_{T}$ and lateral forces $F_{L}$ of the upper slab versus the lateral displacement $s$ in the case of sliding for different $\Theta$ values. Panels (a) and (b) are for $\Theta=0$; panels (c) and (d) are for $\Theta=0.64$ (open circles), $\Theta=0.84$ (solid circles), and $\Theta$ $=1$ (triangles). The loading force $F_{N}=0.03 \mathrm{eV} /$ atom; the sliding speed of the upper slab from right to left, $v=2.5 \mathrm{~m} / \mathrm{sec}$.

Interestingly, the potential energy $V_{T}$ varies linearly in the disordered state that occurs for $4.2<s<5 \AA$. For $5<s$ $<5.25 \AA$ the structure changes from the disordered state to the ordered state ending at the $H$-site registry indicated by $C$ in Fig. 6(a). In spite of the transformation to an ordered state, the minimum of the potential energy $V_{T}$ rises due to the residual defects (such as vacancies, interstitialcies, and partial dislocations); so $V_{T}(C)>V_{T}(A)$. Figure 7 shows the disorder-order structural transformation ${ }^{18}$ for $s=4.5 \AA$ and $s=5.5 \AA$ for $\Theta=0$. The variation of total energy $V_{T}$ is conservative in the period $A \rightarrow B$ or $C \rightarrow D$. However, due to the residual defect formation or structural transformation in the slab, $V_{T}(C)-V_{T}(A) \sim 25 \mathrm{eV}$ and hence the total potential energy undergoes irreversible change. The structural transformation and defect formations are easily recognized in Figs. 7(a) and 7(b). The lateral force $F_{L}$ is obtained from the derivative of $V_{T}$ :

$$
F_{L}(s)=-\frac{\partial V_{T}}{\partial s} .
$$

$F_{L}>0$ is along the $y$ direction, and is in the opposite direction of the external force, which moves the upper slab.

The implementation of the $\mathrm{Xe}$ atoms between two $\mathrm{Ni}(110)$ slabs with coverage $0<\Theta<1$, changes the situation dramatically. This is seen from the corrugation of $V_{T}(s)$ that reduced from $\sim 170 \mathrm{eV}(\Theta=0)$ to $12.5 \mathrm{eV}(\Theta=0.64)$. Under the loading force $F_{N}=0.03 \mathrm{eV} /(\AA$ atom $)$ the Xe atoms prevent the system from any defect formation, except some structural transformation within the lubricant layer. As a result, energy lost is reduced, and so is the average friction force. Initially, the atoms of the upper $\mathrm{Ni}(110)$ slabs are located just above the $\mathrm{Ni}$ atoms of the lower slab. The $T$-site configuration is now a minimum energy configuration owing to the presence of $\mathrm{Xe}$ atoms located at the $H$ sites of both $\mathrm{Ni}$ slabs. By moving the upper slab, the Xe atoms are forced to move in the same direction. This increases $V_{T}$ until a maximum value occurring at the displacement, $s<(n-1) a$ 

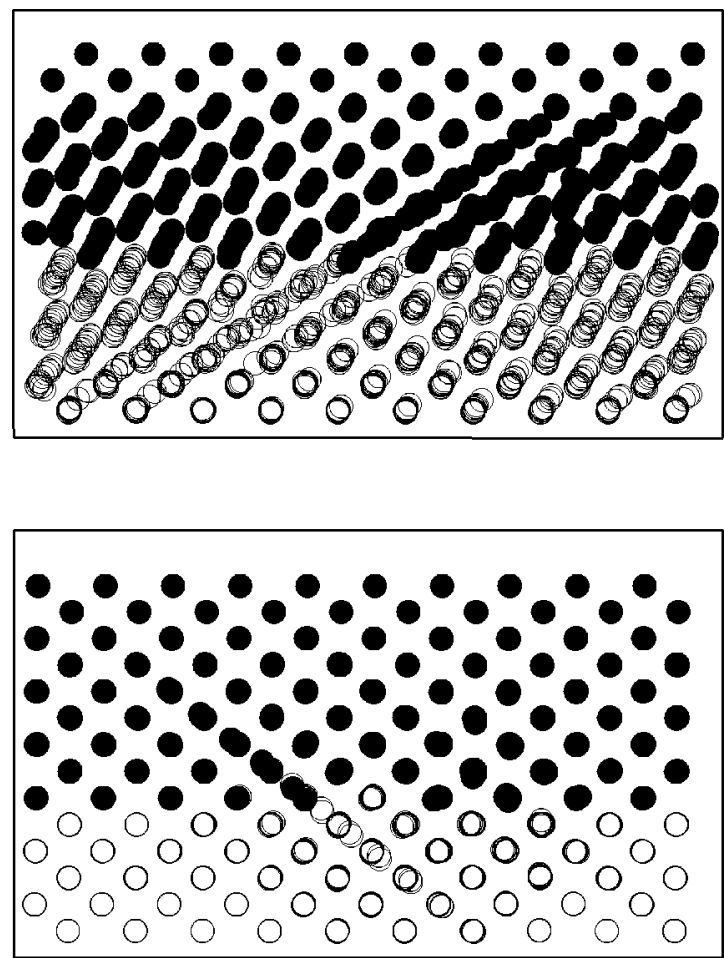

FIG. 7. Snapshots of side views before and after a disorderorder structural transformation for $\Theta=0$. The upper panel is for $s$ $=4.5 \AA$ before the transformation and the lower panel is for $s$ $=5.5 \AA$ after the transformation. The $\mathrm{Ni}$ atoms of the upper (lower) slab are shown by solid (open) circles.

$+a / 2$; thereafter the potential energy is lowered since the Xe atoms move to the displaced $H$ sites of the upper and lower surfaces. As a result, the maximum of $V_{T}$ is decreased to give a local minimum. The maximum change of $V_{T}$ in a period or potential energy corrugation, $\Delta V_{T}$, increases with $\Theta ; \Delta V_{T}$ is $20 \mathrm{eV}, 16 \mathrm{eV}$ and $12 \mathrm{eV}$ for $\Theta=1,0.84$, and 0.64 , respectively. The side and top views of $\Theta=0.84$ are shown in Fig. 8 for different lateral displacements. Once the displacement reaches the plateau region of $V_{T}(s)$ (i.e., $1<s$ $<2 \AA$ ), the upper slab first rises and then attracts part of the $\mathrm{Xe}$ atoms to accommodate at its $H$ site positions. So these Xe atoms are carried with the upper slab for a while. Remaining part of Xe atoms keep their initial positions during sliding (see the middle panels of Fig. 8). By passing the plateau region upon continuing displacement, the $\mathrm{Xe}$ atoms which were attached to the $H$, site positions of the upper slab descend to the similar positions of the lower slab. This way a row of vacancies is created between two domains of Xe atoms, which reduces $\Delta V_{T}$. This is reminiscent of the motion of a line dislocation, which reduces the shear strength. As noted, the situation is rather different for the case of $\Theta=1$, since there is no space to create a row of vacancies. In the plateau of the first period of $V_{T}(s)$ all Xe atoms keep their positions close to the $H$-site registry of the lower slab while the upper slab is sliding over. In contrast to that, in the plateau region of the second period of $V_{T}(s)$ whole Xe layer moves and first pass to the $H$ site position of the upper slab. Then, being attached to it they slide over the lower slab. Note that different behaviors of the Xe layer in two consecutive period of $V_{T}(s)$ are clarified by the variation of the layer- averaged local pressure $P_{L}(s)$. As illustrated in Fig. 9,
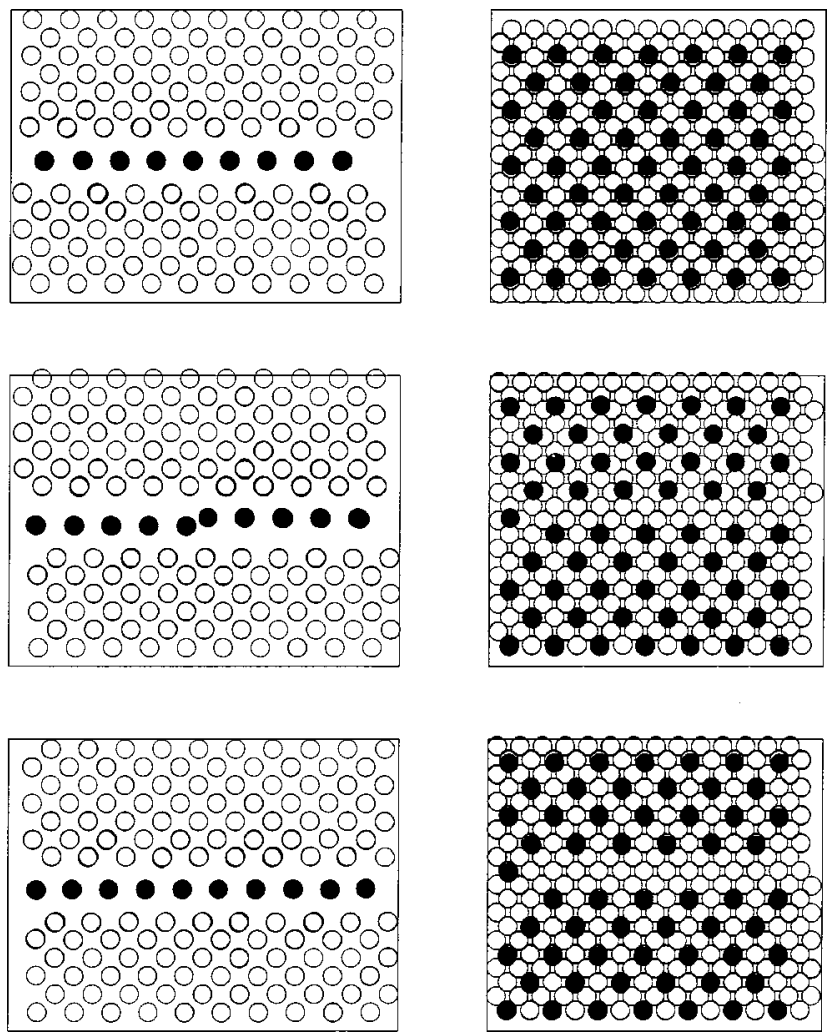

FIG. 8. Snapshots of side and top views of the upper Ni(110) slab moving laterally above the lower $\mathrm{Ni}(110)$ slab with Xe coverage $\Theta=0.84$. Upper, middle, and lower panels correspond to the lateral displacement $s=1.0,2.0$, and $3.0 \AA$ A, respectively.

while the top layer of the lower slab is under relatively stronger expansive pressure in the first period of $V_{T}$, the bottom layer of the upper slab comes under stronger expansive pressure in the subsequent period. Stated differently, the Ni layer that has the higher expansive pressure keeps the Xe atoms. Clearly, this shows that during sliding, the BornOppenheimer surface was modified in such a way that two minima with the same energies occur. If $\Theta<1$, part of the lubricant atoms can move to the $H$-site registry while the others keep their initial positions. This way, vacancies in the lubricant layer propagate. In the full coverage $(\Theta=1)$ case

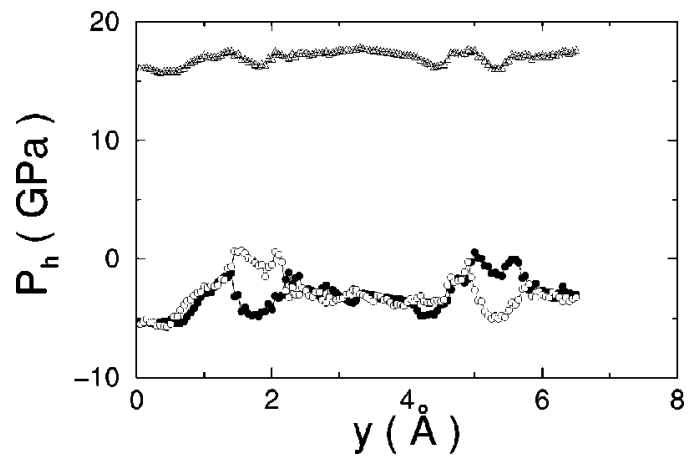

FIG. 9. Layer averaged local hydrostatic pressure $P_{L}$ as function of lateral displacement $s$, in the course of sliding for $\Theta=1$. The pressure values are shown for the bottom layer of the upper slab, the top layer of the lower slab, and the lubricant layer by open circles, solid circles, and triangles, respectively. 
the lubricant atoms either move or keep their place as a whole. Finally we note that since the translation of the upper slab by a lattice constant involves whole Xe atoms, $\Delta V_{T}$ is larger as compared to that for $\Theta<1$. For the same reason $P_{L}$ of the Xe layer depends on the coverage. Since $\Delta V_{T}$ is largest for $\Theta=1, P_{L}$ is expected to be highest among the coverages corresponding to $\Theta \geqslant 0$.

\section{DISCUSSION AND CONCLUSIONS}

Earlier, the lubrication by molecules and atoms were studied by different groups theoretically and experimentally. The prime motivation of these studies has been reducing the friction. Recent publications, as well as the present work, have focused on the atomic processes taking place in the course of the relative motion of objects; hence these studies are relevant for the understanding of energy dissipation mechanisms. The energy dissipation due to the inert-gas atoms physisorbed on the metal substrates has been measured by Krim and his collaborators. ${ }^{19}$ It is argued that the energy is dissipated mainly by electronic ${ }^{20,21}$ and phononic processes; ${ }^{22}$ their share in the total energy lost has been a subject of dispute. Of course, in the systems operating under significant loading force similar to the one treated here the amount of energy transformed into residual defects can be significant. Hot electrons created as a result of friction can transport energy and mediate the dissipation. The mechanism proposed earlier for the energy dissipation ${ }^{21}$ is questioned seriously by the recent work of Dayo et al., ${ }^{23}$ who observed that the friction force due to the motion of a nitrogen layer absorbed on the $\mathrm{Pb}$ film decreases abruptly when the metal substrate is cooled below $T_{c}$.

In atomic scale studies based on molecular dynamics calculations a major part of the dissipated energy $\mathcal{E}_{p}$ is taken from the system in the course of thermalization to a constant temperature. The remaining part of the energy $\mathcal{E}_{d}$ is reimplemented into the system through the structural transformations induced by the relative motion under the loading force $F_{N}$. If $\mathcal{E}_{d}$ is negligible, the dynamic friction constant $\mu_{d}$, averaged over a translation $s$, can be calculated by using $\mathcal{E}_{p}$, which is taken out of the system in the same period of $s$. Here we discuss a phononic mechanism, and present a theoretical model that can provide for an atomic scale analysis of energy dissipation in the boundary lubrication. A similar model used to investigate the energy dissipation in the dry sliding friction of a copper asperity on the copper surface revealed interesting aspects of dissipation process. ${ }^{24} \mathrm{~A}$ nonequilibrium phonon distribution was generated as a result of sudden (or nonadiabatic) motion of atoms. In the present case Ni atoms on the slab surfaces, in particular Xe atoms, are released suddenly from their (metastable) strained state as they overcome the barrier. Let us consider the displacements of $\mathrm{Xe}$ and Ni atoms at the interface $\left\{\mathbf{u}_{i}\right\}$ which correspond to a metastable state $\Psi_{m}$ just before those atoms execute jumps to their stable positions (which is taken as the beginning of one dissipation cycle, $t=0) . \Psi_{m}$ stores energy by increasing the potential energy $\delta V$, which is subtracted from the mechanical energy. As soon as atoms are released from their metastable states, certain vibrational modes are excited and $\delta V$ is transformed into the vibrational energy of the atoms irreversibly. Here $\delta V$ corresponds to $\mathcal{E}_{p}$ occurring during a single cycle seen in Fig. 6(c). Eventually, the phonon distribution of normal modes, $n_{q}$, increases locally. The energy transferred to the vibrational modes cannot be changed back to the mechanical energy, and is dissipated at a certain rate fixed by the temperature and material parameters. The metastable state $\Psi_{m}\left(\mathbf{u}_{i}\right)$ can be projected to the normal modes $\phi_{q}$ :

$$
\Psi_{m}=\sum_{q} a_{q} \phi_{q}
$$

The kinetic energy associated with the excitation of a mode $\phi_{q}$ is equivalent to

$$
\delta V_{q}(t=0)=\frac{1}{2} M \Omega_{q}^{2} a_{q}^{2},
$$

which, at the same time, is equal to $\hbar \Omega_{q}\left(n_{q}+1 / 2\right)$. Here $n_{q}=n\left(\Omega_{q}, T\right)$ stands for Planck's distribution. The excess distribution due to the vibrational excitation is obtained by subtracting the equilibrium distribution corresponding to the ambient temperature $T_{0}, n_{q}^{0}=n_{q}\left(\Omega_{q}, T_{0}\right)$ from $n_{q}$, i.e. $\Delta n_{q}$ $=n_{q}-n_{q}^{0}$. This excess distribution of phonons and hence the energy associated with it are dissipated to the substrates mainly by harmonic coupling. The decay rate of each vibrational mode $\Gamma_{q}$, which is also expressed ${ }^{24,25}$ as $R\left(\Omega_{q}\right) n_{q}$, can be calculated in terms of harmonic coupling parameters, which in turn are estimated by using the recent work of Madsen et $a l .{ }^{26}$ Following our earlier work, ${ }^{24} d \Delta n_{q} / d t=-\Gamma_{q}$ yields the time variation of the phonon distribution, $n_{q}(t)$ $=n_{q}^{0} \exp \left[-R\left(\Omega_{q}\right) t\right]$. Then, the energy dissipation extracted therefrom is

$$
\delta V(t)=\sum_{q} \delta V_{q}(0) \exp \left[-R\left(\Omega_{q}\right) t\right] .
$$

Normally, $\delta V$ is dissipated completely to the samples within one cycle. ${ }^{24}$ We note that in the above analysis it is assumed that both the ambient temperature and normal modes are unaltered in the course of the relative motions of upper and lower slabs. In reality, the normal modes and their level spacing $\Delta \Omega$ vary with $s$ (or with time $t$ ). The ambient temperature $T_{0}$ of finite slabs is also subject to change in the course of relative motion. Therefore, both $T_{0}$ and $\Omega_{q}$ need to be calculated self-consistently.

In conclusion, we examined how a lubricant operates to reduce friction. The important findings of the present work are the following: (i) The corrugation of the total potential energy $\Delta V_{T}$ is reduced dramatically with the Xe coverage. ( $\Delta V_{T}$ is reduced from $173 \mathrm{eV}$ to $12.5 \mathrm{eV}$ by implementing $\mathrm{Xe}$ atoms corresponding to $\Theta=0.64$.) Interestingly, $\Delta V_{T}$ has a minimum value for $\Theta<1 ; \Delta V_{T}$ is very large for $\Theta=0$, but starts to decrease with increasing $\Theta$ and passes through a minimum. The detailed analysis of $\Theta<0.5$ and $1<\Theta<3$ is expected to be also interesting. (ii) Another important feature clarified in this work is the motion of Xe atoms during the motion of the upper slab; Xe atoms jump to the hollow sites of the lower and upper slabs when $s \sim(n-1) a+\frac{1}{2} a$. The sudden motion can be the source of energy lost. (iii) The weak interaction between $\mathrm{Xe}-\mathrm{Ni}$ and $\mathrm{Xe}-\mathrm{Xe}$ and reduced interaction between $\mathrm{Ni}-\mathrm{Ni}$ due to the large radius of $\mathrm{Xe}$ are the prime causes of the reduced energy lost. (iv) Since the resis- 
tance of the lubricant layer is found to be coverage dependent, the study of the effect of normal load on the friction is important. Then, how the kinetic friction constant depends on $F_{N}$ is an interesting question to be answered. (v) A theoretical discussion of the energy dissipation is presented, and a model is proposed that can provide for an atomic scale analysis of phononic energy dissipation in boundary lubrication.

\section{ACKNOWLEDGMENT}

S.C. acknowledges financial support from the Turkish Academy of Sciences.
${ }^{1}$ F.P. Bowden and D. Tabor, Friction and Lubrication (Methuen, London, 1965); E. Rabinowicz, Friction and Wear (Wiley, New York, 1965); J.N. Israelachvili, P.M. McGuiggan, and H.M. Homola, Science 240, 189 (1987); Physics of Sliding Friction, Vol. 311 of NATO Advanced Study Institute, Series E: Applied Science, edited by B. N. J. Persson and E. Tosatti (Kluwer, Dordrecht, 1996).

${ }^{2}$ G. Binnig, C.F. Quate, and Ch. Gerber, Phys. Rev. Lett. 56, 930 (1986).

${ }^{3}$ C.M. Mate, G.M. McClelland, R. Erlandson, and S. Chang, Phys. Rev. Lett. 59, 1942 (1987).

${ }^{4}$ U. Landman, W.D. Luedke, N.A. Burnham, and R.J. Colton, Science 248, 454 (1990).

${ }^{5}$ P.A. Thompson, and M.O. Robbins, Phys. Rev. A 41, 6830 (1990); Science 250, 792 (1990).

${ }^{6}$ A.P. Sutton, and J.B. Pethica, J. Phys.: Condens. Matter 2, 5317 (1990); J.A. Nieminen, A.P. Sutton, and J.B. Pethica, Acta Metall. Mater. 40, 2503 (1992).

${ }^{7}$ M. Cieplak, E.D. Smith, and M.O. Robins, Science 265, 1209 (1994).

${ }^{8}$ M.R. Sørensen, K.W. Jacobsen, and P. Stoltze, Phys. Rev. B 53, 2101 (1996); M.R. Sørensen, K.W. Jacobsen, and H. Jónsson, Phys. Rev. Lett. 77, 5067 (1996).

${ }^{9}$ A. Buldum, and S. Ciraci, Phys. Rev. B 55, 2606 (1997); A. Buldum, S. Ciraci, and Inder P. Batra, ibid. 57, 2468 (1998).

${ }^{10}$ S. Ciraci, E. Tekman, A. Baratoff, and I.P. Batra, Phys. Rev. B 46, 10411 (1992).

${ }^{11}$ H. Yoshizawa, Y.L. Chen, and J. Israelachvili, Wear 168, 161 (1993).

${ }^{12}$ B.N.J. Persson, Phys. Rev. B 55, 8004 (1997).

${ }^{13}$ O.M. Braun, T. Dauxois, and M. Peyrard, Phys. Rev. B 56, 4987 (1997).

${ }^{14}$ J.E. Black, and A. Jansen, Phys. Rev. B 39, 6238 (1989).

${ }^{15}$ A. Buldum, S. Ciraci, and Ş. Erkoç, J. Phys.: Condens. Matter 7, 8487 (1995).

${ }^{16}$ M.S. Daw, and M.I. Baskes, Phys. Rev. B 29, 6443 (1984); S.M. Foiles, M.I. Baskes, and M.S. Daw, ibid. 33, 7983 (1986); M.S. Daw, ibid. 39, 7441 (1989). We used the embedded atom model potentials for $\mathrm{Ni}$ developed in the above works.
${ }^{17}$ For a system of particles under the pair potential interaction $U_{i j}$, the total potential energy is $V_{T}=\Sigma_{i, j} U_{i j} / 2$. In T. Egami, and D. Srolovitz, J. Phys. F 12, 2141 (1982), the atomic level stress for the $i$ th atom is expressed as $\sigma_{i}^{\alpha \beta}=\left(1 / 2 \mathrm{v}_{i}\right) \Sigma_{j}\left(1 / r_{i j}\right)$ $\times\left(d U_{i j} / d r_{i j}\right) r_{i j}^{\alpha} r_{i j}^{\beta}$ where $\mathrm{v}_{i}$ is the atomic volume of the $i$ th atom and $\alpha, \beta$ are the Cartesian components. In the embedded atom model one uses force instead of $d U_{i j} / d r_{i j}$.

${ }^{18}$ Similar structural transformations were observed in the atomic scale simulations of metal nanowires under tensile stress [see, for example, H. Mehrez, and S. Ciraci, Phys. Rev. B 56, 12632 (1997); H. Mehrez, S. Ciraci, C.Y. Fong, and S. Erkoc, J. Phys.: Condens. Matter 9, 10843 (1997)]; and of dry sliding friction of the $\mathrm{Ni}(111)$ tip on the $\mathrm{Cu}(110)$ slab (see Ref. 9). A quantitative analysis of the order-disorder structural transformation can be provided by calculating the structure factor, $S(\mathbf{q})$ $=N^{-1} \int P(\mathbf{r}) e^{i \mathbf{q} \cdot \mathbf{r}} d \mathbf{r}$, by using the autocorrelation function $P(\mathbf{r})=\Sigma_{i, j} \delta\left(\mathbf{r}+\tau_{j}-\tau_{i}\right)$ in terms of the position vectors of the atoms, $\tau_{i}$ and $\tau_{j}$ [see, for example, A. Buldum, and S. Ciraci, Phys. Rev. B 55, 12892 (1997)]. However, a qualitative analysis of the order-disorder structural transformation can be obtained from the analysis of the computer generated snapshots showing the positions of atoms in various stages of the translation.

${ }^{19}$ J. Krim, and A. Widom, Phys. Rev. B 38, 12184 (1988); C. Daly and J. Krim, Phys. Rev. Lett. 76, 803 (1996).

${ }^{20}$ L.S. Levitov, Europhys. Lett. 8, 499 (1989).

${ }^{21}$ B.N.J. Persson, Phys. Rev. B 44, 3277 (1991); B.N.J. Persson, and A.I. Volotkin, J. Chem. Phys. 103, 8679 (1995).

${ }^{22}$ M. Cieplak, E.D. Smith, and M.O. Robins, Science 265, 1209 (1994).

${ }^{23}$ A. Dayo, W. Alnasrallah, and J. Krim, Phys. Rev. Lett. 80, 1690 (1998).

${ }^{24}$ A. Buldum, D. Leitner, and S. Ciraci, Phys. Rev. B 59, 16042 (1999).

${ }^{25}$ G.A. Voth, J. Chem. Phys. 88, 5547 (1988); G.A. Voth, and R.A. Marcus, ibid., 84, 2254 (1986).

${ }^{26}$ D. Madsen, R. Pearman, and M. Gruebele, J. Chem. Phys. 106, 5874 (1997). 